\title{
The modified viscosity implicit rules for uniformly L-Lipschitzian asymptotically pseudocontractive mappings in Banach spaces
}

\author{
Yuanheng Wang*, Chanjuan Pan \\ Department of Mathematics, Zhejiang Normal University, Jinhua, China. \\ Communicated by Y. H. Yao
}

\begin{abstract}
The aim of this paper is to establish the modified viscosity implicit rules for uniformly L-Lipschitzian asymptotically pseudocontractive mappings in Banach spaces. The strong convergence theorems of the rules are proved under certain assumptions imposed on the sequences of parameters. As an application, we apply our main results to solve some variational inequalities in Banach spaces, provided $\mathrm{T}$ is asymptotically regular. Our results extend the previous known results from Hilbert spaces to Banach spaces and from non-expansive mappings to asymptotically pseudocontractive mappings. (C)2017 All rights reserved.
\end{abstract}

Keywords: Viscosity implicit rule, variational inequality, strong convergence, asymptotically pseudo-contractions, Banach space.

2010 MSC: 47H05, 47H09, 47H10, 54H25.

\section{Introduction and preliminaries} by

Let $E$ be a real Banach space. Let $J$ denote the normalized duality mapping from $E$ into $2^{E^{*}}$ defined

$$
J(x)=\left\{f \in E^{*}:\langle x, f\rangle=\|x\|^{2}=\|f\|^{2}\right\}
$$

for all $x \in E$, where $E^{*}$ denotes the dual space of $E$ and $\langle\cdot, \cdot\rangle$ denotes the generalized duality pairing, we denote the single-valued duality mapping by $j$. In a Hilbert space $\mathrm{H}, j$ is the identity mapping.

Let $C$ be a nonempty closed convex subset of $E$. A mapping $f: C \rightarrow C$ is said to be a strict contraction if there exists a constant $\lambda \in(0,1)$ satisfying

$$
\|f(x)-f(y)\| \leqslant \lambda\|x-y\|, \quad \forall x, y \in C .
$$

A mapping $T: C \rightarrow C$ is said to be an asymptotically non-expansive if there exists a sequence $\left\{k_{n}\right\}$ with $k_{\mathrm{n}} \rightarrow 1$ such that

$$
\left\|T^{n} x-T^{n} y\right\| \leqslant k_{n}\|x-y\|, \quad \forall x, y \in C \text {. }
$$

\footnotetext{
*Corresponding author

Email addresses: yhwang@zjnu.cn (Yuanheng Wang), cjpanzjnu@163.com (Chanjuan Pan)
}

doi:10.22436/jnsa.010.04.24 
A mapping $\mathrm{T}: \mathrm{C} \rightarrow \mathrm{C}$ is said to be an asymptotically pseudocontraction in Banach spaces if there exists a sequence $\left\{k_{n}\right\}$ with $k_{n} \rightarrow 1$ and $j(x-y) \in J(x-y)$ for which the following inequality holds

$$
\left\langle T^{n} x-T^{n} y, j(x-y)\right\rangle \leqslant k_{n}\|x-y\|^{2}, \quad \forall x, y \in C, n \geqslant 1 .
$$

A mapping $T: C \rightarrow C$ is said to be uniform L-Lipschitzian if there exists some $L>0$ such that

$$
\left\|T^{n} x-T^{n} y\right\| \leqslant L\|x-y\|, \quad \forall x, y \in C, \quad \forall n \geqslant 1 .
$$

It is easy to see that if $T$ is an asymptotically nonexpansive mapping, then it is both asymptotically pseudocontractive and uniformly L-Lipschitzian. The converse is not true in general.

Convergence analysis for fixed point of nonexpansive mapping and asymptotically nonexpansive mapping using the viscosity iterative algorithms have been studied extensively by many authors, see $[1-8,10-12,14,15]$ and the references therein. In 2000, moudafi [7] proved the strong convergence theorem for nonexpansive mappings in real Hilbert spaces. In 2004, Xu [11] studied the following viscosity approximation methods of nonexpansive mapping in a uniformly smooth Banach:

$$
x_{n+1}=\alpha_{n} f\left(x_{n}\right)+\left(1-\alpha_{n}\right) T x_{n}, \quad n \geqslant 0,
$$

where $\mathrm{f}$ is contraction and $\left\{\alpha_{n}\right\}$ is a sequence in $\{0,1\}$. In 2015, Xu et al. [12] applied the viscosity technique to the implicit midpoint rule for nonexpansive mappings in Hilbert spaces. They proved that the iterative process defined by

$$
x_{n+1}=\alpha_{n} f\left(x_{n}\right)+\left(1-\alpha_{n}\right) T\left(\frac{x_{n}+x_{n+1}}{2}\right), n \geqslant 0,
$$

converges in norm to a fixed point $\mathrm{q}$ of $\mathrm{T}$, which solves the variational inequality:

$$
\langle(I-f) q, x-q\rangle \geqslant 0, x \in F(T) .
$$

Further, Yao et al. [14] introduced the following semi-implicit midpoint method:

$$
x_{n+1}=\alpha_{n} x_{n}+\beta_{n} f\left(x_{n}\right)+\gamma_{n} T\left(\frac{x_{n}+x_{n+1}}{2}\right), n \geqslant 0 .
$$

In 2016, Yu et al. [15] extended the work of Yao et al. [14] and gave the following generalized viscosity implicit rules of nonexpansive mappings in Hilbert spaces:

$$
x_{n+1}=\alpha_{n} f\left(x_{n}\right)+\beta_{n} x_{n}+\gamma_{n} T\left(s_{n} x_{n}+\left(1-s_{n}\right) x_{n+1}\right), \quad n \geqslant 0,
$$

which converges strongly to a fixed point of $\mathrm{T}$ under certain assumptions imposed on the sequences of parameters.

In this paper, motivated by the above mentioned works, we introduce a modified viscosity implicit rules for a uniformly L-Lipschitzian asymptotically pseudocontractive mappings in Banach spaces. Then, under suitable assumptions imposed on the parameters, we establish strong convergence theorems for asymptotical pseudocontractions in Banach spaces. As an application, we apply our main results to solve the variational inequality problems in Banach spaces. Therefore, we extend the main results of Yu et al. (see [15]) from Hilbert spaces to Banach spaces when $T$ is an asymptotically nonexpansive respective asymptotically pseudocontractive map. Further, some other results are also improved (see $[1,5,7,8,11$, $12,14])$.

We shall make use of the following lemmas.

Lemma 1.1 ([11]). Assume $\left\{a_{n}\right\}$ is a sequence of nonnegative real numbers such that

$$
a_{n+1} \leqslant\left(1-\alpha_{n}\right) a_{n}+\delta_{n}, \quad n \geqslant 0,
$$

where $\left\{\alpha_{n}\right\}$ is a sequence in $(0,1)$ and $\left\{\delta_{n}\right\}$ is a sequence in $R$ such that 
(i) $\sum_{n=0}^{\infty} \alpha_{n}=\infty$;

(ii) $\limsup _{n \rightarrow \infty} \frac{\delta_{n}}{\alpha_{n}} \leqslant 0$ or $\sum_{n=1}^{\infty}\left|\delta_{n}\right|<\infty$.

Then, $\lim _{n \rightarrow \infty} a_{n}=0$.

Lemma 1.2 ([9]). Let $\mathrm{E}$ be a reflexive smooth Banach space with a weakly sequential continuous duality mapping J. Let $\mathrm{C}$ be a nonempty bounded and closed convex subset of $\mathrm{E}$ and $\mathrm{T}: \mathrm{C} \rightarrow \mathrm{C}$ be a uniformly L-Lipschitzian and asymptotical pseudocontraction. Then $\mathrm{I}-\mathrm{T}$ is demiclosed at zero, where $\mathrm{I}$ is the identical mapping, i.e., if $\mathrm{x}_{\mathrm{n}} \rightarrow$ $x$ weakly and $x_{n}-T x_{n} \rightarrow 0$ strongly, then $x \in F(T)$.

Lemma 1.3 ([14]). Let $\left\{x_{n}\right\}$ and $\left\{y_{n}\right\}$ be bounded sequence in a Banach space $E$ and $\left\{\beta_{n}\right\}$ be a sequence in $[0,1]$ with $0<\liminf _{n \rightarrow \infty} \beta_{n} \leqslant \limsup _{n \rightarrow \infty} \beta_{n}$. Suppose that $x_{n+1}=\left(1-\beta_{n}\right)+\beta_{n} y_{n}$ for all $n \geqslant 0$ and

$$
\limsup _{n \rightarrow \infty}\left(\left\|y_{n+1}-y_{n}\right\|-\left\|x_{n+1}-x_{n}\right\|\right) \leqslant 0
$$

Then $\lim _{n \rightarrow \infty}\left\|y_{n}-x_{n}\right\|=0$.

\section{Main results}

Theorem 2.1. Let $\mathrm{E}$ be a reflexive smooth Banach space with a weakly sequential continuous duality mapping J, $\mathrm{C}$ a nonempty bounded and closed convex subset of $\mathrm{E}$, and Let $\mathrm{T}: \mathrm{C} \rightarrow \mathrm{C}$ be a uniformly L-Lipschitzian asymptotically pseudocontractive mapping with a sequence $\left\{\mathrm{k}_{\mathrm{n}}\right\}$ such that $\mathrm{F}(\mathrm{T}) \neq \emptyset$ and $\mathrm{f}: \mathrm{C} \rightarrow \mathrm{C}$ be a contraction with coefficient $\lambda \in(0,1)$. Pick any $x_{0} \in \mathrm{C}$, let $x_{\mathrm{n}}$ be a sequence generated by

$$
x_{n+1}=\alpha_{n} x_{n}+\beta_{n} f\left(x_{n}\right)+\gamma_{n} T^{n}\left(\xi_{n} x_{n}+\left(1-\xi_{n}\right) x_{n+1}\right),
$$

where $\left\{\alpha_{n}\right\},\left\{\beta_{n}\right\},\left\{\gamma_{n}\right\},\left\{\xi_{n}\right\} \subset(0,1)$, satisfying the following conditions:

(i) $\alpha_{n}+\beta_{n}+\gamma_{n}=1, \lim _{n \rightarrow \infty} \beta_{n}=0, \gamma_{n}=\eta \beta_{n}, 0<\eta<\frac{1-\lambda}{L-1}$;

(ii) $0<\liminf _{n \rightarrow \infty} \alpha_{n} \leqslant \limsup _{n \rightarrow \infty} \alpha_{n}<1, \lim _{n \rightarrow \infty} \beta_{n}=0, \lim _{n \rightarrow \infty}\left|\alpha_{n+1}-\alpha_{n}\right|=0, \lim _{n \rightarrow \infty} \mid \beta_{n+1}-$ $\beta_{n} \mid=0$;

(iii) $\sum_{n=0}^{\infty} \beta_{n}=\infty, 0<\xi_{n} \leqslant \xi_{n+1}<1, \gamma_{n}\left(1-\xi_{n}\right)<\frac{1}{L}$;

(iv) $\lim _{n \rightarrow \infty}\left\|T^{n+1} x-T^{n} x\right\|=0, x \in C^{\prime}$, where $C^{\prime}$ is any bounded subset of $C$ for all $n \geqslant 0$.

Then $\left\{x_{n}\right\}$ converges strongly to a fixed point $\mathrm{p}$ of the asymptotically pseudocontractive mapping $T$, which solves the variational inequality:

$$
\langle(I-f) p, j(p-y)\rangle \leqslant 0, \quad \forall y \in F(T) .
$$

Proof. We divide the proof into five steps.

Step 1: First, we show that $\left\{x_{n}\right\}$ is bounded.

Take $p \in F(T)$ arbitrarily, we have

$$
\begin{aligned}
\left\|x_{n+1}-p\right\| & =\left\|\alpha_{n} x_{n}+\beta_{n} f\left(x_{n}\right)+\gamma_{n} T^{n}\left(\xi_{n} x_{n}+\left(1-\xi_{n}\right) x_{n+1}\right)-p\right\| \\
& =\left\|\alpha_{n}\left(x_{n}-p\right)+\beta_{n}\left(f\left(x_{n}\right)-f(p)\right)+\beta_{n}(f(p)-p)+\gamma_{n}\left(T^{n}\left(\xi_{n} x_{n}+\left(1-\xi_{n}\right) x_{n+1}\right)-p\right)\right\| \\
& \leqslant \alpha_{n}\left\|x_{n}-p\right\|+\beta_{n}\left\|f\left(x_{n}\right)-f(p)\right\|+\beta_{n}\|f(p)-p\|+\gamma_{n}\left\|T^{n}\left(\xi_{n} x_{n}+\left(1-\xi_{n}\right) x_{n+1}\right)-p\right\| \\
& \leqslant \alpha_{n}\left\|x_{n}-p\right\|+\beta_{n} \lambda\left\|x_{n}-p\right\|+\beta_{n}\|f(p)-p\|+\gamma_{n} L\left\|\left(\xi_{n} x_{n}+\left(1-\xi_{n}\right) x_{n+1}\right)-p\right\| \\
& \leqslant \alpha_{n}\left\|x_{n}-p\right\|+\beta_{n} \lambda\left\|x_{n}-p\right\|+\beta_{n}\|f(p)-p\|+\gamma_{n} L \xi_{n}\left\|x_{n}-p\right\|+\gamma_{n} L\left(1-\xi_{n}\right)\left\|x_{n+1}-p\right\| \\
& =\left(\alpha_{n}+\lambda \beta_{n}+\gamma_{n} L \xi_{n}\right)\left\|x_{n}-p\right\|+\beta_{n}\|f(p)-p\|+\gamma_{n} L\left(1-\xi_{n}\right)\left\|x_{n+1}-p\right\|,
\end{aligned}
$$

which implies that

$$
\left[1-\gamma_{n} L\left(1-\xi_{n}\right)\right]\left\|x_{n+1}-p\right\| \leqslant\left(\alpha_{n}+\lambda \beta_{n}+\gamma_{n} L \xi_{n}\right)\left\|x_{n}-p\right\|+\beta_{n}\|f(p)-p\| .
$$


Since $\gamma_{n}, 1-\xi_{n} \in(0,1), \gamma_{n}\left(1-\xi_{n}\right)<\frac{1}{L}$, we get $1-\gamma_{n} L\left(1-\xi_{n}\right)>0$. By (2.2) and condition (i), it then follows that

$$
\begin{aligned}
\left\|x_{n+1}-p\right\| & \leqslant \frac{\alpha_{n}+\lambda \beta_{n}+\gamma_{n} L \xi_{n}}{1-\gamma_{n} L\left(1-\xi_{n}\right)}\left\|x_{n}-p\right\|+\frac{\beta_{n}}{1-\gamma_{n} L\left(1-\xi_{n}\right)}\|f(p)-p\| \\
& =\left[1-\frac{1-\alpha_{n}-\lambda \beta_{n}-\gamma_{n} L}{1-\gamma_{n} L\left(1-\xi_{n}\right)}\right]\left\|x_{n}-p\right\|+\frac{\beta_{n}}{1-\gamma_{n} L\left(1-\xi_{n}\right)}\|f(p)-p\| \\
& =\left[1-\frac{\beta_{n}(1-\lambda)-\gamma_{n}(L-1)}{1-\gamma_{n} L\left(1-\xi_{n}\right)}\right]\left\|x_{n}-p\right\|+\frac{\beta_{n}}{1-\gamma_{n} L\left(1-\xi_{n}\right)}\|f(p)-p\| \\
& \leqslant\left[1-\frac{\beta_{n}[1-\lambda-\eta(L-1)]}{1-\gamma_{n} L\left(1-\xi_{n}\right)}\right]\left\|x_{n}-p\right\|+\frac{\beta_{n}[1-\lambda-\eta(L-1)]}{1-\gamma_{n} L\left(1-\xi_{n}\right)} \frac{\|f(p)-p\|}{1-\lambda-\eta(L-1)} .
\end{aligned}
$$

Consequently, we get

$$
\left\|x_{n+1}-p\right\| \leqslant \max \left\{\left\|x_{n}-p\right\|, \frac{1}{1-\lambda-\eta(L-1)}\|f(p)-p\|\right\}, \quad \forall n \geqslant 0
$$

By induction we readily obtain

$$
\left\|x_{n}-p\right\| \leqslant \max \left\{\left\|x_{0}-p\right\|, \frac{1}{1-\lambda-\eta(L-1)}\|f(p)-p\|\right\}, \quad \forall n \geqslant 0 .
$$

Hence we obtain that $x_{n}$ is bounded. It turns out that $f\left(x_{n}\right), T^{n}\left(\xi_{n} x_{n}+\left(1-\xi_{n}\right) x_{n+1}\right)$ are also bounded.

Step 2: $\lim _{n \rightarrow \infty}\left\|x_{n+1}-x_{n}\right\|=0$. To see this we set $y_{n}=\frac{x_{n+1}-\alpha_{n} x_{n}}{1-\alpha_{n}}$, for all $n \geqslant 0$. Thus we have

$$
\begin{aligned}
y_{n+1}-y_{n}= & \frac{x_{n+2}-\alpha_{n+1} x_{n+1}}{1-\alpha_{n+1}}-\frac{x_{n+1}-\alpha_{n} x_{n}}{1-\alpha_{n}} \\
= & \frac{\beta_{n+1} f\left(x_{n+1}\right)+\gamma_{n+1} T^{n+1}\left(\xi_{n+1} x_{n+1}+\left(1-\xi_{n+1}\right) x_{n+2}\right)}{1-\alpha_{n+1}} \\
& -\frac{\beta_{n} f\left(x_{n}\right)+\gamma_{n} T^{n}\left(\xi_{n} x_{n}+\left(1-\xi_{n}\right) x_{n+1}\right)}{1-\alpha_{n}} \\
= & \frac{\beta_{n+1} f\left(x_{n+1}\right)+\left(1-\alpha_{n+1}-\beta_{n+1}\right) T^{n+1}\left(\xi_{n+1} x_{n+1}+\left(1-\xi_{n+1}\right) x_{n+2}\right)}{1-\alpha_{n+1}} \\
& -\frac{\beta_{n} f\left(x_{n}\right)+\left(1-\alpha_{n}-\beta_{n}\right) T^{n}\left(\xi_{n} x_{n}+\left(1-\xi_{n}\right) x_{n+1}\right)}{1-\alpha_{n}} \\
= & \frac{\beta_{n+1}}{1-\alpha_{n+1}}\left[f\left(x_{n+1}\right)-f\left(x_{n}\right)\right]+\left(\frac{\beta_{n+1}}{1-\alpha_{n+1}}-\frac{\beta_{n}}{1-\alpha_{n}}\right) f\left(x_{n}\right) \\
& -\left(\frac{\beta_{n+1}}{1-\alpha_{n+1}}-\frac{\beta_{n}}{1-\alpha_{n}}\right) T^{n}\left(\xi_{n} x_{n}+\left(1-\xi_{n}\right) x_{n+1}\right) \\
& -\frac{\beta_{n+1}}{1-\alpha_{n+1}}\left[T^{n+1}\left(\xi_{n+1} x_{n+1}+\left(1-\xi_{n+1}\right) x_{n+2}\right)-T^{n}\left(\xi_{n} x_{n}+\left(1-\xi_{n}\right) x_{n+1}\right)\right] \\
& +\left[T^{n+1}\left(\xi_{n+1} x_{n+1}+\left(1-\xi_{n+1}\right) x_{n+2}\right)-T^{n}\left(\xi_{n} x_{n}+\left(1-\xi_{n}\right) x_{n+1}\right)\right] \\
= & \frac{\beta_{n+1}}{1-\alpha_{n+1}}\left[f\left(x_{n+1}\right)-f\left(x_{n}\right)\right]+\left(\frac{\beta_{n+1}}{1-\alpha_{n+1}}-\frac{\beta_{n}}{1-\alpha_{n}}\right)\left[f\left(x_{n}\right)-T^{n}\left(\xi_{n} x_{n}+\left(1-\xi_{n}\right) x_{n+1}\right)\right] \\
& -\frac{\beta_{n+1}}{1-\alpha_{n+1}}\left[T^{n+1}\left(\xi_{n+1} x_{n+1}+\left(1-\xi_{n+1}\right) x_{n+2}\right)-T^{n}\left(\xi_{n} x_{n}+\left(1-\xi_{n}\right) x_{n+1}\right)\right] \\
& +\left[T^{n+1}\left(\xi_{n+1} x_{n+1}+\left(1-\xi_{n+1}\right) x_{n+2}\right)-T^{n}\left(\xi_{n} x_{n}+\left(1-\xi_{n}\right) x_{n+1}\right)\right] \\
= & \frac{\beta_{n+1}}{1-\alpha_{n+1}}\left[f\left(x_{n+1}\right)-f\left(x_{n}\right)\right]+\left(\frac{\beta_{n+1}}{1-\alpha_{n+1}}-\frac{\beta_{n}}{1-\alpha_{n}}\right)\left[f\left(x_{n}\right)-T^{n}\left(\xi_{n} x_{n}+\left(1-\xi_{n}\right) x_{n+1}\right)\right] \\
& =1
\end{aligned}
$$




$$
\begin{aligned}
& +\left(1-\frac{\beta_{n+1}}{1-\alpha_{n+1}}\right)\left[T^{n+1}\left(\xi_{n+1} x_{n+1}+\left(1-\xi_{n+1}\right) x_{n+2}\right)-T^{n}\left(\xi_{n+1} x_{n+1}+\left(1-\xi_{n+1}\right) x_{n+2}\right)\right] \\
& +\left(1-\frac{\beta_{n+1}}{1-\alpha_{n+1}}\right)\left[T^{n}\left(\xi_{n+1} x_{n+1}+\left(1-\xi_{n+1}\right) x_{n+2}\right)-T^{n}\left(\xi_{n} x_{n}+\left(1-\xi_{n}\right) x_{n+1}\right)\right] .
\end{aligned}
$$

It follows that

$$
\begin{aligned}
\left\|y_{n+1}-y_{n}\right\| \leqslant & \frac{\lambda \beta_{n+1}}{1-\alpha_{n+1}}\left\|x_{n+1}-x_{n}\right\|+\left|\frac{\beta_{n+1}}{1-\alpha_{n+1}}-\frac{\beta_{n}}{1-\alpha_{n}}\right| M+\sup _{x \in C^{\prime}}\left\|T^{n+1} x-T^{n} x\right\| \\
& +\left(1-\frac{\beta_{n+1}}{1-\alpha_{n+1}}\right) L\left\|\xi_{n+1} x_{n+1}+\left(1-\xi_{n+1}\right) x_{n+2}-\left(\xi_{n} x_{n}+\left(1-\xi_{n}\right) x_{n+1}\right)\right\| \\
\leqslant & \frac{\lambda \beta_{n+1}}{1-\alpha_{n+1}}\left\|x_{n+1}-x_{n}\right\|+\left|\frac{\beta_{n+1}}{1-\alpha_{n+1}}-\frac{\beta_{n}}{1-\alpha_{n}}\right| M+\sup _{x \in C^{\prime}}\left\|T^{n+1} x-T^{n} x\right\| \\
& +\left(1-\frac{\beta_{n+1}}{1-\alpha_{n+1}}\right) L\left\|\xi_{n}\left(x_{n+1}-x_{n}\right)+\left(1-\xi_{n+1}\right)\left(x_{n+2}-x_{n+1}\right)\right\| \\
\leqslant & \frac{\lambda \beta_{n+1}}{1-\alpha_{n+1}}\left\|x_{n+1}-x_{n}\right\|+\left|\frac{\beta_{n+1}}{1-\alpha_{n+1}}-\frac{\beta_{n}}{1-\alpha_{n}}\right| M+\sup _{x \in C^{\prime}}\left\|T^{n+1} x-T^{n} x\right\| \\
& +\left(1-\frac{\beta_{n+1}}{1-\alpha_{n+1}}\right) L\left[\xi_{n}\left\|x_{n+1}-x_{n}\right\|+\left(1-\xi_{n+1}\right)\left\|x_{n+2}-x_{n+1}\right\|\right],
\end{aligned}
$$

where $C^{\prime}$ contains sequence $\left\{\xi_{n} x_{n}+\left(1-\xi_{n}\right) x_{n+1}\right\}$ and $M>0$ is a constant such that

$$
\begin{aligned}
M \geqslant\left\{\sup _{n \geqslant 0}\left\|x_{n}-T^{n+1}\left(\xi_{n} x_{n}+\left(1-\xi_{n}\right) x_{n+1}\right)\right\|, \sup _{n \geqslant 0} \| f\left(x_{n}\right)-T^{n+1}\left(\xi_{n} x_{n}+\left(1-\xi_{n}\right) x_{n+1} \|,\right.\right. & \\
& \sup _{n \geqslant 0} \| f\left(x_{n}\right)-T^{n}\left(\xi_{n} x_{n}+\left(1-\xi_{n}\right) x_{n+1} \|\right\} .
\end{aligned}
$$

From (2.1), we have

$$
\begin{aligned}
\left\|x_{n+2}-x_{n+1}\right\|= & \| \alpha_{n+1} x_{n+1}+\beta_{n+1} f\left(x_{n+1}\right)+\gamma_{n+1} T^{n+1}\left(\xi_{n+1} x_{n+1}+\left(1-\xi_{n+1}\right) x_{n+2}\right) \\
& -\alpha_{n} x_{n}-\beta_{n} f\left(x_{n}\right)-\gamma_{n} T^{n}\left(\xi_{n} x_{n}+\left(1-\xi_{n}\right) x_{n+1}\right) \| \\
= & \| \alpha_{n+1}\left(x_{n+1}-x_{n}\right)+\left(\alpha_{n+1}-\alpha_{n}\right) x_{n}+\beta_{n+1}\left(f\left(x_{n+1}\right)-f\left(x_{n}\right)\right)+\left(\beta_{n+1}-\beta_{n}\right) f\left(x_{n}\right) \\
& +\gamma_{n+1}\left[T^{n+1}\left(\xi_{n+1} x_{n+1}+\left(1-\xi_{n+1}\right) x_{n+2}\right)-T^{n+1}\left(\xi_{n} x_{n}+\left(1-\xi_{n}\right) x_{n+1}\right)\right] \\
& +\left(\gamma_{n+1}-\gamma_{n}\right) T^{n+1}\left(\xi_{n} x_{n}+\left(1-\xi_{n}\right) x_{n+1}\right) \\
& +\gamma_{n}\left[T^{n+1}\left(\xi_{n} x_{n}+\left(1-\xi_{n}\right) x_{n+1}\right)-T^{n}\left(\xi_{n} x_{n}+\left(1-\xi_{n}\right) x_{n+1}\right)\right] \| \\
= & \| \alpha_{n+1}\left(x_{n+1}-x_{n}\right)+\left(\alpha_{n+1}-\alpha_{n}\right) x_{n}+\beta_{n+1}\left(f\left(x_{n+1}\right)-f\left(x_{n}\right)\right)+\left(\beta_{n+1}-\beta_{n}\right) f\left(x_{n}\right) \\
& +\gamma_{n+1}\left[T^{n+1}\left(\xi_{n+1} x_{n+1}+\left(1-\xi_{n+1}\right) x_{n+2}\right)-T^{n+1}\left(\xi_{n} x_{n}+\left(1-\xi_{n}\right) x_{n+1}\right)\right] \\
& -\left[\left(\alpha_{n+1}-\alpha_{n}\right)+\left(\beta_{n+1}-\beta_{n}\right)\right] T^{n+1}\left(\xi_{n} x_{n}+\left(1-\xi_{n}\right) x_{n+1}\right) \\
& +\gamma_{n}\left[T^{n+1}\left(\xi_{n} x_{n}+\left(1-\xi_{n}\right) x_{n+1}\right)-T^{n}\left(\xi_{n} x_{n}+\left(1-\xi_{n}\right) x_{n+1}\right)\right] \| \\
= & \| \alpha_{n+1}\left(x_{n+1}-x_{n}\right)+\left(\alpha_{n+1}-\alpha_{n}\right)\left[x_{n}-T^{n+1}\left(\xi_{n} x_{n}+\left(1-\xi_{n}\right) x_{n+1}\right)\right] \\
& +\left(\beta_{n+1}-\beta_{n}\right)\left[f\left(x_{n}\right)-T^{n+1}\left(\xi_{n} x_{n}+\left(1-\xi_{n}\right) x_{n+1}\right)\right]+\beta_{n+1}\left(f\left(x_{n+1}\right)-f\left(x_{n}\right)\right) \\
& +\gamma_{n+1}\left[T^{n+1}\left(\xi_{n+1} x_{n+1}+\left(1-\xi_{n+1}\right) x_{n+2}\right)-T^{n+1}\left(\xi_{n} x_{n}+\left(1-\xi_{n}\right) x_{n+1}\right)\right] \\
& +\gamma_{n}\left[T^{n+1}\left(\xi_{n} x_{n}+\left(1-\xi_{n}\right) x_{n+1}\right)-T^{n}\left(\xi_{n} x_{n}+\left(1-\xi_{n}\right) x_{n+1}\right)\right] \| \\
\leqslant & \alpha_{n+1}\left\|x_{n+1}-x_{n}\right\|+\left|\alpha_{n+1}-\alpha_{n}\right|\left\|x_{n}-T^{n+1}\left(\xi_{n} x_{n}+\left(1-\xi_{n}\right) x_{n+1}\right)\right\| \\
& +\left|\beta_{n+1}-\beta_{n}\right| \| f\left(x_{n}\right)-T^{n+1}\left(\xi_{n} x_{n}+\left(1-\xi_{n}\right) x_{n+1}\left\|+\lambda \beta_{n+1}\right\| x_{n+1}-x_{n} \|\right. \\
& +\gamma_{n+1} L\left\|\xi_{n+1} x_{n+1}+\left(1-\xi_{n+1}\right) x_{n+2}-\xi_{n} x_{n}-\left(1-\xi_{n}\right) x_{n+1}\right\|
\end{aligned}
$$




$$
\begin{aligned}
& +\gamma_{n}\left\|T^{n+1}\left(\xi_{n} x_{n}+\left(1-\xi_{n}\right) x_{n+1}\right)-T^{n}\left(\xi_{n} x_{n}+\left(1-\xi_{n}\right) x_{n+1}\right)\right\| \\
\leqslant & \alpha_{n+1}\left\|x_{n+1}-x_{n}\right\|+\left|\alpha_{n+1}-\alpha_{n}\right| M+\left|\beta_{n+1}-\beta_{n}\right| M+\lambda \beta_{n+1}\left\|x_{n+1}-x_{n}\right\| \\
& +\gamma_{n+1} L\left\|\left(1-\xi_{n+1}\right)\left(x_{n+2}-x_{n+1}\right)+\xi_{n}\left(x_{n+1}-x_{n}\right)\right\| \\
& +\gamma_{n}\left\|T^{n+1}\left(\xi_{n} x_{n}+\left(1-\xi_{n}\right) x_{n+1}\right)-T^{n}\left(\xi_{n} x_{n}+\left(1-\xi_{n}\right) x_{n+1}\right)\right\| \\
\leqslant & \left(\alpha_{n+1}+\lambda \beta_{n+1}+\gamma_{n+1} L \xi_{n}\right)\left\|x_{n+1}-x_{n}\right\|+\gamma_{n+1} L\left(1-\xi_{n+1}\right)\left\|x_{n+2}-x_{n+1}\right\| \\
& +\left(\left|\alpha_{n+1}-\alpha_{n}\right|+\left|\beta_{n+1}-\beta_{n}\right|\right) M \\
& +\gamma_{n}\left\|T^{n+1}\left(\xi_{n} x_{n}+\left(1-\xi_{n}\right) x_{n+1}\right)-T^{n}\left(\xi_{n} x_{n}+\left(1-\xi_{n}\right) x_{n+1}\right)\right\| \\
\leqslant & \left(\alpha_{n+1}+\lambda \beta_{n+1}+\gamma_{n+1} L \xi_{n}\right)\left\|x_{n+1}-x_{n}\right\|+\gamma_{n+1} L\left(1-\xi_{n+1}\right)\left\|x_{n+2}-x_{n+1}\right\| \\
& +\left(\left|\alpha_{n+1}-\alpha_{n}\right|+\left|\beta_{n+1}-\beta_{n}\right|\right) M+\sup _{x \in C^{\prime}}\left\|T^{n+1} x-T^{n} x\right\| .
\end{aligned}
$$

It turns out that

$$
\begin{aligned}
{\left[1-\gamma_{n+1} L\left(1-\xi_{n+1}\right)\right]\left\|x_{n+2}-x_{n+1}\right\| \leqslant } & \left(\alpha_{n+1}+\lambda \beta_{n+1}+\gamma_{n+1} L \xi_{n}\right)\left\|x_{n+1}-x_{n}\right\| \\
& +\left(\left|\alpha_{n+1}-\alpha_{n}\right|+\left|\beta_{n+1}-\beta_{n}\right|\right) M+\sup _{x \in C^{\prime}} \|\left[T^{n+1} x-T^{n} x \|,\right.
\end{aligned}
$$

and moreover,

$$
\begin{aligned}
& \left\|x_{n+2}-x_{n+1}\right\| \\
& \leqslant \frac{\alpha_{n+1}+\lambda \beta_{n+1}+\gamma_{n+1} L \xi_{n}}{1-\gamma_{n+1} L\left(1-\xi_{n+1}\right)}\left\|x_{n+1}-x_{n}\right\|+\frac{M}{1-\gamma_{n+1} L\left(1-\xi_{n+1}\right)}\left(\left|\alpha_{n+1}-\alpha_{n}\right|+\left|\beta_{n+1}-\beta_{n}\right|\right) \\
& +\frac{1}{1-\gamma_{n+1} L\left(1-\xi_{n+1}\right)} \sup _{x \in C^{\prime}}\left\|T^{n+1} x-T^{n} x\right\| \\
& =\left[1-\frac{\beta_{n+1}(1-\lambda)+\gamma_{n+1} L\left(\xi_{n+1}-\xi_{n}\right)-\gamma_{n+1}(L-1)}{1-\gamma_{n+1} L\left(1-\xi_{n+1}\right)}\right]\left\|x_{n+1}-x_{n}\right\| \\
& +\frac{M}{1-\gamma_{n+1} L\left(1-\xi_{n+1}\right)}\left(\left|\alpha_{n+1}-\alpha_{n}\right|+\left|\beta_{n+1}-\beta_{n}\right|\right)+\frac{1}{1-\gamma_{n+1} L\left(1-\xi_{n+1}\right)} \sup _{x \in C^{\prime}}\left\|T^{n+1} x-T^{n} x\right\| \\
& \leqslant\left[1-\frac{\beta_{n+1}[1-\lambda-\eta(L-1)]+\gamma_{n+1} L\left(\xi_{n+1}-\xi_{n}\right)}{1-\gamma_{n+1} L\left(1-\xi_{n+1}\right)}\right]\left\|x_{n+1}-x_{n}\right\| \\
& +\frac{M}{1-\gamma_{n+1} L\left(1-\xi_{n+1}\right)}\left(\left|\alpha_{n+1}-\alpha_{n}\right|+\left|\beta_{n+1}-\beta_{n}\right|\right)+\frac{1}{1-\gamma_{n+1} L\left(1-\xi_{n+1}\right)} \sup _{x \in C^{\prime}}\left\|T^{n+1} \chi-T^{n} x\right\| .
\end{aligned}
$$

Substitute (2.4) into (2.3), we get

$$
\begin{aligned}
\left\|y_{n+1}-y_{n}\right\| \leqslant & {\left[\frac{\lambda \beta_{n+1}}{1-\alpha_{n+1}}+\left(1-\frac{\beta_{n+1}}{1-\alpha_{n+1}}\right) L \xi_{n}+\left(1-\frac{\beta_{n+1}}{1-\alpha_{n+1}}\right) L\left(1-\xi_{n+1}\right)\right]\left\|x_{n+1}-x_{n}\right\| } \\
& +\frac{\gamma_{n+1} L\left(1-\xi_{n+1}\right) M}{\left(1-\alpha_{n+1}\right)\left[1-\gamma_{n+1} L\left(1-\xi_{n+1}\right)\right]}\left(\left|\alpha_{n+1}-\alpha_{n}\right|+\left|\beta_{n+1}-\beta_{n}\right|\right)+\sup _{x \in C^{\prime}}\left\|T^{n+1} x-T^{n} x\right\| \\
& +\frac{\gamma_{n+1} L\left(1-\xi_{n+1}\right)}{\left(1-\alpha_{n+1}\right)\left[1-\gamma_{n+1} L\left(1-\xi_{n+1}\right)\right]} \sup _{x \in C^{\prime}}\left\|T^{n+1} x-T^{n} x\right\|+\left|\frac{\beta_{n+1}}{1-\alpha_{n+1}}-\frac{\beta_{n}}{1-\alpha_{n}}\right| M \\
= & \frac{\lambda \beta_{n+1}+\gamma_{n+1} L \xi_{n}+\gamma_{n+1} L\left(1-\xi_{n+1}\right)}{1-\alpha_{n+1}}\left\|x_{n+1}-x_{n}\right\|+\left|\frac{\beta_{n+1}}{1-\alpha_{n+1}}-\frac{\beta_{n}}{1-\alpha_{n}}\right| M \\
& +\frac{1}{\left(1-\alpha_{n+1}\right)\left[1-\gamma_{n+1} L\left(1-\xi_{n+1}\right)\right]} \sup _{x \in C^{\prime}}\left\|T^{n+1} x-T^{n} x\right\| \\
& +\frac{\gamma_{n+1} L\left(1-\xi_{n+1}\right) M}{\left(1-\alpha_{n+1}\right)\left[1-\gamma_{n+1} L\left(1-\xi_{n+1}\right)\right]}\left(\left|\alpha_{n+1}-\alpha_{n}\right|+\left|\beta_{n+1}-\beta_{n}\right|\right) \\
\leqslant & \frac{\lambda \beta_{n+1}+\gamma_{n+1} L}{1-\alpha_{n+1}}\left\|x_{n+1}-x_{n}\right\|+\left|\frac{\beta_{n+1}}{1-\alpha_{n+1}}-\frac{\beta_{n}}{1-\alpha_{n}}\right| M
\end{aligned}
$$




$$
\begin{aligned}
& +\frac{1}{\left(1-\alpha_{n+1}\right)\left[1-\gamma_{n+1} L\left(1-\xi_{n+1}\right)\right]} \sup _{x \in \mathrm{C}^{\prime}}\left\|T^{n+1} x-T^{n} x\right\| \\
& +\frac{\gamma_{n+1} L\left(1-\xi_{n+1}\right) M}{\left(1-\alpha_{n+1}\right)\left[1-\gamma_{n+1} L\left(1-\xi_{n+1}\right)\right]}\left(\left|\alpha_{n+1}-\alpha_{n}\right|+\left|\beta_{n+1}-\beta_{n}\right|\right) \\
& =\left[1-\frac{(1-\lambda) \beta_{n+1}-\gamma_{n+1}(L-1)}{1-\alpha_{n+1}}\right]\left\|x_{n+1}-x_{n}\right\|+\left|\frac{\beta_{n+1}}{1-\alpha_{n+1}}-\frac{\beta_{n}}{1-\alpha_{n}}\right| M \\
& +\frac{1}{\left(1-\alpha_{n+1}\right)\left[1-\gamma_{n+1} L\left(1-\xi_{n+1}\right)\right]} \sup _{x \in C^{\prime}}\left\|T^{n+1} x-T^{n} x\right\| \\
& +\frac{\gamma_{n+1} L\left(1-\xi_{n+1}\right) M}{\left(1-\alpha_{n+1}\right)\left[1-\gamma_{n+1} L\left(1-\xi_{n+1}\right)\right]}\left(\left|\alpha_{n+1}-\alpha_{n}\right|+\left|\beta_{n+1}-\beta_{n}\right|\right) \\
& =\left[1-\frac{[1-\lambda-\eta(L-1)] \beta_{n+1}}{1-\alpha_{n+1}}\right]\left\|x_{n+1}-x_{n}\right\|+\left|\frac{\beta_{n+1}}{1-\alpha_{n+1}}-\frac{\beta_{n}}{1-\alpha_{n}}\right| M \\
& +\frac{1}{\left(1-\alpha_{n+1}\right)\left[1-\gamma_{n+1} L\left(1-\xi_{n+1}\right)\right]} \sup _{x \in C^{\prime}}\left\|T^{n+1} x-T^{n} x\right\| \\
& +\frac{\gamma_{n+1} \mathrm{~L}\left(1-\xi_{n+1}\right) M}{\left(1-\alpha_{n+1}\right)\left[1-\gamma_{n+1} \mathrm{~L}\left(1-\xi_{n+1}\right)\right]}\left(\left|\alpha_{n+1}-\alpha_{n}\right|+\left|\beta_{n+1}-\beta_{n}\right|\right) \text {. }
\end{aligned}
$$

By conditions (i) and (ii), we can get

$$
\limsup _{n \rightarrow \infty}\left(\left\|y_{n+1}-y_{n}\right\|-\left\|x_{n+1}-x_{n}\right\|\right) \leqslant 0
$$

Applying Lemma 1.3, we have

$$
\lim _{n \rightarrow \infty}\left\|y_{n}-x_{n}\right\|=0
$$

Note that

$$
y_{n}-x_{n}=\frac{x_{n+1}-x_{n}}{1-\alpha_{n}}
$$

So we obtain

$$
\lim _{n \rightarrow \infty}\left\|x_{n+1}-x_{n}\right\|=0
$$

Step 3: Next we prove that $\lim _{n \rightarrow \infty}\left\|x_{n}-T x_{n}\right\|=0$. In fact, we observe

$$
\begin{aligned}
& \left\|x_{n+1}-T^{n}\left(\xi_{n} x_{n}+\left(1-\xi_{n}\right) x_{n+1}\right)\right\| \\
& =\left\|\alpha_{n} x_{n}+\beta_{n} f\left(x_{n}\right)-\alpha_{n} T^{n}\left(\xi_{n} x_{n}+\left(1-\xi_{n}\right) x_{n+1}\right)-\beta_{n} T^{n}\left(\xi_{n} x_{n}+\left(1-\xi_{n}\right) x_{n+1}\right)\right\| \\
& =\left\|\alpha_{n}\left[x_{n}-T^{n}\left(\xi_{n} x_{n}+\left(1-\xi_{n}\right) x_{n+1}\right)\right]+\beta_{n}\left[f\left(x_{n}\right)-T^{n}\left(\xi_{n} x_{n}+\left(1-\xi_{n}\right) x_{n+1}\right)\right]\right\| \\
& \leqslant \alpha_{n}\left\|x_{n}-x_{n+1}\right\|+\alpha_{n}\left\|x_{n+1}-T^{n}\left(\xi_{n} x_{n}+\left(1-\xi_{n}\right) x_{n+1}\right)\right\|+\beta_{n}\left\|f\left(x_{n}\right)-T^{n}\left(\xi_{n} x_{n}+\left(1-\xi_{n}\right) x_{n+1}\right)\right\|,
\end{aligned}
$$

which implies that

$$
\left(1-\alpha_{n}\right)\left\|x_{n+1}-T^{n}\left(\xi_{n} x_{n}+\left(1-\xi_{n}\right) x_{n+1}\right)\right\| \leqslant \alpha_{n}\left\|x_{n}-x_{n+1}\right\|+\beta_{n}\left\|f\left(x_{n}\right)-T^{n}\left(\xi_{n} x_{n}+\left(1-\xi_{n}\right) x_{n+1}\right)\right\| .
$$

That is

$$
\left\|x_{n+1}-T^{n}\left(\xi_{n} x_{n}+\left(1-\xi_{n}\right) x_{n+1}\right)\right\| \leqslant \frac{\alpha_{n}}{1-\alpha_{n}}\left\|x_{n}-x_{n+1}\right\|+\frac{\beta_{n}}{1-\alpha_{n}}\left\|f\left(x_{n}\right)-T^{n}\left(\xi_{n} x_{n}+\left(1-\xi_{n}\right) x_{n+1}\right)\right\| .
$$

By conditions (i), (ii), and applying Step 2, we get

$$
\left\|x_{n+1}-T^{n}\left(\xi_{n} x_{n}+\left(1-\xi_{n}\right) x_{n+1}\right)\right\| \rightarrow 0,(n \rightarrow \infty) .
$$


And, moreover, we have

$$
\begin{aligned}
\left\|x_{n}-T^{n} x_{n}\right\| & =\left\|x_{n}-x_{n+1}+x_{n+1}-T^{n}\left(\xi_{n} x_{n}+\left(1-\xi_{n}\right) x_{n+1}\right)+T^{n}\left(\xi_{n} x_{n}+\left(1-\xi_{n}\right) x_{n+1}\right)-T^{n} x_{n}\right\| \\
& \leqslant\left\|x_{n}-x_{n+1}\right\|+\left\|x_{n+1}-T^{n}\left(\xi_{n} x_{n}+\left(1-\xi_{n}\right) x_{n+1}\right)\right\|+\left\|T^{n}\left(\xi_{n} x_{n}+\left(1-\xi_{n}\right) x_{n+1}\right)-T^{n} x_{n}\right\| \\
& \leqslant\left\|x_{n}-x_{n+1}\right\|+\left\|x_{n+1}-T^{n}\left(\xi_{n} x_{n}+\left(1-\xi_{n}\right) x_{n+1}\right)\right\|+L\left\|\xi_{n} x_{n}+\left(1-\xi_{n}\right) x_{n+1}-x_{n}\right\| \\
& =\left\|x_{n}-x_{n+1}\right\|+\left\|x_{n+1}-T^{n}\left(\xi_{n} x_{n}+\left(1-\xi_{n}\right) x_{n+1}\right)\right\|+L\left(1-\xi_{n}\right)\left\|x_{n+1}-x_{n}\right\| \\
& =\left(1+L\left(1-\xi_{n}\right)\right)\left\|x_{n}-x_{n+1}\right\|+\left\|x_{n+1}-T^{n}\left(\xi_{n} x_{n}+\left(1-\xi_{n}\right) x_{n+1}\right)\right\| .
\end{aligned}
$$

In view of Step 2 and (2.5), we have

$$
\left\|x_{n}-T^{n} x_{n}\right\| \rightarrow 0, \quad n \rightarrow \infty
$$

Since T is uniformly L-Lipschitzian, we derive

$$
\begin{aligned}
\left\|x_{n}-T x_{n}\right\| & =\left\|x_{n}-T^{n} x_{n}+T^{n} x_{n}-T x_{n}\right\| \\
& \leqslant\left\|x_{n}-T^{n} x_{n}\right\|+\left\|T^{n} x_{n}-T x_{n}\right\| \\
& \leqslant\left\|x_{n}-T^{n} x_{n}\right\|+L\left\|T^{n-1} x_{n}-x_{n}\right\| \\
& \leqslant\left\|x_{n}-T^{n} x_{n}\right\|+L\left\|T^{n-1} x_{n}-T^{n-1} x_{n-1}+T^{n-1} x_{n-1}-x_{n-1}+x_{n-1}-x_{n}\right\| \\
& \leqslant\left\|x_{n}-T^{n} x_{n}\right\|+L\left\|T^{n-1} x_{n}-T^{n-1} x_{n-1}\right\|+L\left\|T^{n-1} x_{n-1}-x_{n-1}\right\|+L\left\|x_{n-1}-x_{n}\right\| \\
& \leqslant\left\|x_{n}-T^{n} x_{n}\right\|+L^{2}\left\|x_{n}-x_{n-1}\right\|+L\left\|T^{n-1} x_{n-1}-x_{n-1}\right\|+L\left\|x_{n-1}-x_{n}\right\| \\
& \leqslant\left\|x_{n}-T^{n} x_{n}\right\|+L\left\|T^{n} x_{n-1}-x_{n-1}\right\|+\left(L^{2}+L\right)\left\|x_{n-1}-x_{n}\right\| .
\end{aligned}
$$

By Step 2 and (2.6), we have

$$
\left\|x_{n}-T x_{n}\right\| \rightarrow 0, n \rightarrow \infty .
$$

Step 4: We claim that $\limsup _{n \rightarrow \infty}\left\langle(I-f) p, j\left(p-x_{n}\right)\right\rangle \leqslant 0$.

Since $x_{n}$ is bounded and $C$ is a reflexive Banach space, there exists a subsequence of $x_{n}$ which converges weakly to $u$, we assume that $x_{n_{k}} \rightarrow u$. And

$$
\lim _{k \rightarrow \infty}\left\langle(I-f) p, j\left(p-x_{n_{k}}\right)\right\rangle=\limsup _{n \rightarrow \infty}\left\langle(I-f) p, j\left(p-x_{n}\right)\right\rangle .
$$

Since $C$ is a smooth Banach space, it follows from Step 3 that $\lim _{k \rightarrow \infty}\left\|x_{n_{k}}-T x_{n_{k}}\right\|=0$. From Lemma 1.2, we have $u \in F(T)$. On the other hand, since $p \in F(T)$ satisfies

$$
\langle(I-f) p, j(p-u)\rangle \leqslant 0, \quad \forall u \in F(T),
$$

by weakly sequential continuous duality mapping, we have

$$
\limsup _{n \rightarrow \infty}\left\langle(I-f) p, j\left(p-x_{n}\right)\right\rangle=\lim _{k \rightarrow \infty}\left\langle(I-f) p, j\left(p-x_{n_{k}}\right)\right\rangle=\langle(I-f) p, j(p-u)\rangle \leqslant 0 .
$$

Step 5: Finally, we show that $x_{n}$ converges strongly to $p \in F(T)$. We get

$$
\begin{aligned}
\left\|x_{n+1}-p\right\|^{2}= & \left\langle\alpha_{n} x_{n}+\beta_{n} f\left(x_{n}\right)+\gamma_{n} T^{n}\left(\xi_{n} x_{n}+\left(1-\xi_{n}\right) x_{n+1}\right)-p, j\left(x_{n+1}-p\right)\right\rangle \\
= & \alpha_{n}\left\langle x_{n}-p, j\left(x_{n+1}-p\right)\right\rangle+\beta_{n}\left\langle f\left(x_{n}\right)-p, j\left(x_{n+1}-p\right)\right\rangle \\
& +\gamma_{n}\left\langle T^{n}\left(\xi_{n} x_{n}+\left(1-\xi_{n}\right) x_{n+1}\right)-p, j\left(x_{n+1}-p\right)\right\rangle \\
\leqslant & \alpha_{n}\left\langle x_{n}-p, j\left(x_{n+1}-p\right)\right\rangle+\beta_{n}\left\langle f\left(x_{n}\right)-f(p), j\left(x_{n+1}-p\right)\right\rangle \\
& +\beta_{n}\left\langle f(p)-p, j\left(x_{n+1}-p\right)\right\rangle+\gamma_{n}\left\langle T^{n}\left(\xi_{n} x_{n}+\left(1-\xi_{n}\right) x_{n+1}\right)-p, j\left(x_{n+1}-p\right)\right\rangle \\
\leqslant & \alpha_{n}\left\|x_{n}-p\right\|\left\|x_{n+1}-p\right\|+\beta_{n} \lambda\left\|x_{n}-p\right\|\left\|x_{n+1}-p\right\| \\
& +\beta_{n}\left\langle f(p)-p, j\left(x_{n+1}-p\right)\right\rangle+\gamma_{n} L\left\|\xi_{n} x_{n}+\left(1-\xi_{n}\right) x_{n+1}-p\right\|\left\|x_{n+1}-p\right\|
\end{aligned}
$$




$$
\begin{aligned}
\leqslant & \alpha_{n}\left\|x_{n}-p\right\|\left\|x_{n+1}-p\right\|+\beta_{n} \lambda\left\|x_{n}-p\right\|\left\|x_{n+1}-p\right\|+\beta_{n}\left\langle f(p)-p, j\left(x_{n+1}-p\right)\right\rangle \\
& +\gamma_{n} L \xi_{n}\left\|x_{n}-p\right\|\left\|x_{n+1}-p\right\|+\gamma_{n} L\left(1-\xi_{n}\right)\left\|x_{n+1}-p\right\|^{2} \\
= & {\left[\alpha_{n}+\beta_{n} \lambda+\gamma_{n} L \xi_{n}\right]\left\|x_{n}-p\right\|\left\|x_{n+1}-p\right\|+\gamma_{n} L\left(1-\xi_{n}\right)\left\|x_{n+1}-p\right\|^{2} } \\
& +\beta_{n}\left\langle f(p)-p, j\left(x_{n+1}-p\right)\right\rangle \\
\leqslant & \frac{\alpha_{n}+\beta_{n} \lambda+\gamma_{n} L \xi_{n}}{2}\left\|x_{n}-p\right\|^{2}+\frac{\alpha_{n}+\beta_{n} \lambda+\gamma_{n} L \xi_{n}}{2}\left\|x_{n+1}-p\right\|^{2} \\
& +\gamma_{n} L\left(1-\xi_{n}\right)\left\|x_{n+1}-p\right\|^{2}+\beta_{n}\left\langle f(p)-p, j\left(x_{n+1}-p\right)\right\rangle \\
= & \frac{\alpha_{n}+\beta_{n} \lambda+\gamma_{n} L \xi_{n}}{2}\left\|x_{n}-p\right\|^{2}+\frac{\alpha_{n}+\beta_{n} \lambda+\gamma_{n} L\left(2-\xi_{n}\right)}{2}\left\|x_{n+1}-p\right\|^{2} \\
& +\beta_{n}\left\langle f(p)-p, j\left(x_{n+1}-p\right)\right\rangle,
\end{aligned}
$$

which implies

$$
\left[1-\frac{\alpha_{n}+\beta_{n} \lambda+\gamma_{n} L\left(2-\xi_{n}\right)}{2}\right]\left\|x_{n+1}-p\right\|^{2} \leqslant \frac{\alpha_{n}+\beta_{n} \lambda+\gamma_{n} L \xi_{n}}{2}\left\|x_{n}-p\right\|^{2}+\beta_{n}\left\langle f(p)-p, j\left(x_{n+1}-p\right)\right\rangle .
$$

That is

$$
\begin{aligned}
\left\|x_{n+1}-p\right\|^{2} \leqslant & \frac{\alpha_{n}+\beta_{n} \lambda+\gamma_{n} L \xi_{n}}{2-\alpha_{n}-\lambda \beta_{n}-\gamma_{n} L\left(2-\xi_{n}\right)}\left\|x_{n+1}-p\right\|^{2} \\
& +\frac{2 \beta_{n}}{2-\alpha_{n}-\lambda \beta_{n}-\gamma_{n} L\left(2-\xi_{n}\right)}\left\langle f(p)-p, j\left(x_{n+1}-p\right)\right\rangle \\
= & {\left[1-\frac{2\left(1-\alpha_{n}-\lambda \beta_{n}-\gamma_{n} L\right)}{2-\alpha_{n}-\lambda \beta_{n}-\gamma_{n} L\left(2-\xi_{n}\right)}\right]\left\|x_{n+1}-p\right\|^{2} } \\
& +\frac{2 \beta_{n}}{2-\alpha_{n}-\lambda \beta_{n}-\gamma_{n} L\left(2-\xi_{n}\right)}\left\langle f(p)-p, j\left(x_{n+1}-p\right)\right\rangle .
\end{aligned}
$$

Let

$$
a_{n}=\frac{2\left(1-\alpha_{n}-\lambda \beta_{n}-\gamma_{n} L\right)}{2-\alpha_{n}-\lambda \beta_{n}-\gamma_{n} L\left(2-\xi_{n}\right)}
$$

We have

$$
a_{n} \geqslant 1-\alpha_{n}-\lambda \beta_{n}-\gamma_{n} L=\beta_{n}(1-\lambda)-\gamma_{n}(L-1) \geqslant(1-\lambda) \beta_{n}
$$

We claim that $\sum_{n=0}^{\infty} \alpha_{n}=\infty$. By Step 4, we get $\langle(I-f) p, j(p-y)\rangle \leqslant 0, \quad \forall y \in F(T)$. Apply Lemma 1.1 to (2.7), we conclude that $\lim _{n \rightarrow \infty}\left\|x_{n}-p\right\|=0$. This finishes the proof.

Theorem 2.2. Let $\mathrm{E}$ be a reflexive smooth Banach space with a weakly sequential continuous duality mapping J, $\mathrm{C} a$ nonempty bounded and closed convex subset of $\mathrm{E}$, and Let $\mathrm{T}: \mathrm{C} \rightarrow \mathrm{C}$ be an asymptotically nonexpansive mapping with a sequence $\left\{k_{n}\right\}$ such that $\mathrm{F}(\mathrm{T}) \neq \emptyset$ and $\mathrm{f}: \mathrm{C} \rightarrow \mathrm{C}$ be a contraction with coefficient $\lambda \in(0,1)$. Pick any $\mathrm{x}_{0} \in \mathrm{C}$, let $\mathrm{x}_{\mathrm{n}}$ be a sequence generated by

$$
x_{n+1}=\alpha_{n} x_{n}+\beta_{n} f\left(x_{n}\right)+\gamma_{n} T^{n}\left(\xi_{n} x_{n}+\left(1-\xi_{n}\right) x_{n+1}\right),
$$

where $\left\{\alpha_{n}\right\},\left\{\beta_{n}\right\},\left\{\gamma_{n}\right\},\left\{\xi_{n}\right\} \subset(0,1)$, satisfying the following conditions:

(i) $\alpha_{n}+\beta_{n}+\gamma_{n}=1, \lim _{n \rightarrow \infty} \beta_{n}=0, \gamma_{n}=\eta \beta_{n}, 0<\eta<\frac{1-\lambda}{k_{n}-1}$;

(ii) $0<\liminf _{n \rightarrow \infty} \alpha_{n} \leqslant \limsup _{n \rightarrow \infty} \alpha_{n}<1, \lim _{n \rightarrow \infty}\left|\alpha_{n+1}-\alpha_{n}\right|=0, \lim _{n \rightarrow \infty}\left|\beta_{n+1}-\beta_{n}\right|=0$;

(iii) $\sum_{n=0}^{\infty} \beta_{n}=\infty, 0<\xi_{n} \leqslant \xi_{n+1}<1, \gamma_{n}\left(1-\xi_{n}\right)<\frac{1}{k_{n}}$;

(iv) $\lim _{n \rightarrow \infty}\left\|T^{n+1} x-T^{n} x\right\|=0, x \in C^{\prime}$, where $C^{\prime}$ is any bounded subset of $C$ for all $n \geqslant 0$. 
Then $\left\{x_{n}\right\}$ converges strongly to a fixed point $p$ of the asymptotically pseudocontractive mapping $T$, which solves the variational inequality:

$$
\langle(I-f) p, j(p-y)\rangle \leqslant 0, \quad \forall y \in F(T) .
$$

Theorem 2.3. Let $\mathrm{E}$ be a reflexive smooth Banach space with a weakly sequential continuous duality mapping J, C a nonempty bounded and closed convex subset of $\mathrm{E}$, and Let $\mathrm{T}: \mathrm{C} \rightarrow \mathrm{C}$ be an asymptotically nonexpansive mapping with a sequence $\left\{k_{n}\right\}$ such that $\mathrm{F}(\mathrm{T}) \neq \emptyset$ and $\mathrm{f}: \mathrm{C} \rightarrow \mathrm{C}$ be a contraction with coefficient $\lambda \in(0,1)$. Pick any $\mathrm{x}_{0} \in \mathrm{C}$, let $\mathrm{x}_{\mathrm{n}}$ be a sequence generated by

$$
x_{n+1}=\alpha_{n} x_{n}+\beta_{n} f\left(x_{n}\right)+\gamma_{n} T^{n}\left(\xi_{n} x_{n}+\left(1-\xi_{n}\right) x_{n+1}\right),
$$

where $\left\{\alpha_{n}\right\},\left\{\beta_{n}\right\},\left\{\gamma_{n}\right\},\left\{\xi_{n}\right\} \subset(0,1)$, satisfying the following conditions:

(i) $\alpha_{n}+\beta_{n}+\gamma_{n}=1, \lim _{n \rightarrow \infty} \beta_{n}=0, k_{n}-1=o\left(\beta_{n}\right)$;

(ii) $0<\liminf _{n \rightarrow \infty} \alpha_{n} \leqslant \limsup _{n \rightarrow \infty} \alpha_{n}<1, \lim _{n \rightarrow \infty}\left|\alpha_{n+1}-\alpha_{n}\right|=0, \lim _{n \rightarrow \infty}\left|\beta_{n+1}-\beta_{n}\right|=0$;

(iii) $\sum_{n=0}^{\infty} \beta_{n}=\infty, 0<\xi_{n} \leqslant \xi_{n+1}<1, \gamma_{n}\left(1-\xi_{n}\right)<\frac{1}{k_{n}}$;

(iv) $\lim _{n \rightarrow \infty}\left\|T^{n+1} x-T^{n} x\right\|=0, x \in C^{\prime}$, where $C^{\prime}$ is any bounded subset of $C$ for all $n \geqslant 0$.

Then $\left\{x_{n}\right\}$ converges strongly to a fixed point $p$ of the asymptotically pseudocontractive mapping $T$, which solves the variational inequality:

$$
\langle(I-f) p, j(p-y)\rangle \leqslant 0, \quad \forall y \in F(T) .
$$

Theorem 2.4. Let $\mathrm{E}$ be a reflexive smooth Banach space with a weakly sequential continuous duality mapping J, C a nonempty bounded and closed convex subset of $\mathrm{E}$, and Let $\mathrm{T}: \mathrm{C} \rightarrow \mathrm{C}$ be a uniformly L-Lipschitzian asymptotically pseudocontractive mapping with a sequence $\left\{k_{n}\right\}$ such that $\mathrm{F}(\mathrm{T}) \neq \emptyset$. Pick any $\mathrm{x}_{0} \in \mathrm{C}$, let $\mathrm{x}_{\mathrm{n}}$ be a sequence generated by

$$
x_{n+1}=\alpha_{n} x_{n}+\beta_{n} u+\gamma_{n} T^{n}\left(\xi_{n} x_{n}+\left(1-\xi_{n}\right) x_{n+1}\right),
$$

where $\left\{\alpha_{n}\right\},\left\{\beta_{n}\right\},\left\{\gamma_{n}\right\},\left\{\xi_{n}\right\} \subset(0,1)$, satisfying the following conditions:

(i) $\alpha_{n}+\beta_{n}+\gamma_{n}=1, \lim _{n \rightarrow \infty} \beta_{n}=0, \gamma_{n}=\eta \beta_{n}, 0<\eta<\frac{1-\lambda}{L-1}$;

(ii) $0<\liminf _{n \rightarrow \infty} \alpha_{n} \leqslant \limsup _{n \rightarrow \infty} \alpha_{n}<1, \lim _{n \rightarrow \infty}\left|\alpha_{n+1}-\alpha_{n}\right|=0, \lim _{n \rightarrow \infty}\left|\beta_{n+1}-\beta_{n}\right|=0$;

(iii) $\sum_{n=0}^{\infty} \beta_{n}=\infty, 0<\xi_{n} \leqslant \xi_{n+1}<1, \gamma_{n}\left(1-\xi_{n}\right)<\frac{1}{L}$;

(iv) $\lim _{n \rightarrow \infty}\left\|T^{n+1} x-T^{n} x\right\|=0, x \in C^{\prime}$, where $C^{\prime}$ is any bounded subset of $C$ for all $n \geqslant 0$.

Then $\left\{x_{n}\right\}$ converges strongly to a fixed point $\mathrm{p}$ of the asymptotically pseudocontractive mapping $T$.

Proof. In this case the map $f: C \rightarrow C$ defined by $f(x)=u, \forall x \in C$ is a strict contraction with constant $\lambda=$ 0 . The proof follows from Theorem 2.1 above.

Remark 2.5. Since every nonexpansive mapping is asymptotically nonexpansive and an asymptotically nonexpansive mapping is both asymptotically pseudocontractive and uniformly L-Lipschitzian, in Theorems 2.1 and 2.2, if $\mathrm{T}$ is a nonexpansive mapping in Hilbert spaces, then it is the main results of Yu et al. [15]. Thus, Theorem 2.1 improves and extends the Yu et al.'s theorem in several respects and improves some other results (see $[1,5,7,8,11,12,14]$ ).

Remark 2.6. We note that, in Theorems 2.2 and 2.3, We can choose condition $k_{n}-1=o\left(\beta_{n}\right)$ replaced the requirement $\gamma_{n}=\eta \beta_{n}, 0<\eta<\frac{1-\lambda}{k_{n}-1}$. However, the proof is similar to Theorem 2.1 above.

Remark 2.7. In Theorem 2.4, if $\xi_{n}=0$, then $\left\{x_{n}\right\}$ converges strongly to a fixed point of $\mathrm{T}$. It is the main results of Yao et al. [13].

\section{Acknowledgment}

The authors would like to thank editors and referees for many useful comments and suggestions for the improvement of the article. This work was supported by the National Natural Science Foundation of China (Grant no. 11671365) and the Natural Science Foundation of Zhejiang Province (Grant no. LY14A010011). 


\section{References}

[1] G. Cai, S.-Q. Bu, Hybrid algorithm for generalized mixed equilibrium problems and variational inequality problems and fixed point problems, Comput. Math. Appl., 62 (2011), 4772-4782. 1, 2.5

[2] L.-C. Ceng, S. Al-Homidan, Q. H. Ansari, J.-C. Yao, An iterative scheme for equilibrium problems and fixed point problems of strict pseudo-contraction mappings, J. Comput. Appl. Math., 223 (2009), 967-974.

[3] F. Gu, A new hybrid iteration method for a finite family of asymptotically nonexpansive mappings in Banach spaces, Fixed Point Theory Appl., 2013 (2013), 10 pages.

[4] A. Kangtunyakarn, S. Suantai, A new mapping for finding common solutions of equilibrium problems and fixed point problems of finite family of nonexpansive mappings, Nonlinear Anal., 71 (2009), 4448-4460.

[5] Y.-F. Ke, C.-F. Ma, A new relaxed extragradient-like algorithm for approaching common solutions of generalized mixed equilibrium problems, a more general system of variational inequalities and a fixed point problem, Fixed Point Theory Appl., 2013 (2013), 21 pages. 1, 2.5

[6] J. Lou, L.-J. Zhang, Z. He, Viscosity approximation methods for asymptotically nonexpansive mappings, Appl. Math. Comput., 203 (2008), 171-177.

[7] A. Moudafi, Viscosity approximation methods for fixed-points problems, J. Math. Anal. Appl., 241 (2000), 46-55. 1, 2.5

[8] X.-L. Qin, Y. J. Cho, S. M. Kang, Viscosity approximation methods for generalized equilibrium problems and fixed point problems with applications, Nonlinear Anal., 72 (2010), 99-112. 1, 2.5

[9] Y.-H. Wang, Y.-H. Xia, Strong convergence for asymptotically pseudocontractions with the demiclosedness principle in Banach spaces, Fixed Point Theory Appl., 2012 (2012), 8 pages. 1.2

[10] Y. H. Wang, L. C. Zeng, Convergence of generalized projective modified iteration methods in Banach spaces, (Chinese) Chinese Ann. Math. Ser. A, 30 (2009), 55-62. 1

[11] H.-K. Xu, Viscosity approximation methods for nonexpansive mappings, J. Math. Anal. Appl., 298 (2004), 279-291. 1, $1.1,2.5$

[12] H.-K. Xu, M. A. Alghamdi, N. Shahzad, The viscosity technique for the implicit midpoint rule of nonexpansive mappings in Hilbert spaces, Fixed Point Theory Appl., 2015 (2015), 12 pages. 1, 2.5

[13] Y.-H. Yao, Y.-C. Liou, R.-D. Chen, Strong convergence of an iterative algorithm for pseudocontractive mapping in Banach spaces, Nonlinear Anal., 67 (2007), 3311-3317. 2.7

[14] Y.-H. Yao, N. Shahzad, Y.-C. Liou, Modified semi-implicit midpoint rule for nonexpansive mappings, Fixed Point Theory Appl., 2015 (2015), 15 pages. 1, 1.3, 2.5

[15] Y.-L. Yu, C.-F. Wen, A modified iterative algorithm for nonexpansive mappings, J. Nonlinear Sci. Appl., 9 (2016), 37193726. 1, 2.5 